\title{
ASCORBIC ACID PROTECTS AGAINST RESTRAINT STRESS-INDUCED MEMORY DEFICITS IN WISTAR RATS
}

\author{
Raju Suresh Kumar, ${ }^{\mathrm{I}}$ Sareesh Naduvil Narayanan, ${ }^{\mathrm{I}}$ Satheesha Nayak ${ }^{\mathrm{II}}$
}

doi: $10.1590 / \mathrm{S} 1807-59322009001200012$

Kumar RS, Narayanan SN, S Nayak. Ascorbic acid protects against restraint stress-induced memory deficits in wistar rats. Clinics. 2009;64(12):1211-7.

OBJECTIVE: Chronic stress has been shown to cause oxidative damage in the central nervous system. Although stress-induced impairments in learning and memory have been studied extensively, very few studies have investigated possible ways to prevent their ill effects. The present work was designed to study the protective effects of ascorbic acid in memory loss induced by chronic restraint stress.

METHODS: Adult male Wistar rats were designated into the following groups: (i) Normal control, (ii) Ascorbic acid treatment, (iii) Vehicle control, (iv) Restraint stress, (v) Restraint stress + vehicle, and (vi) Restraint stress + ascorbic acid treatment. At the end of 21 days, animals of all groups were subjected to memory tests using Morris water maze and passive avoidance apparatus. Then, the results obtained were compared between the experimental groups.

RESULTS: Rats exposed to restraint stress alone and those pretreated with vehicle solution before restrained stress showed deficits in learning and impaired memory retention in the memory tests when compared to animals in other experimental groups. Animals pretreated with ascorbic acid before restraining showed significant improvement in memory retention in the same memory tests.

CONCLUSIONS: Results of this study suggest the possibility of using ascorbic acid as a dietary supplement to prevent stressinduced memory impairments.

KEYWORDS: Ascorbic acid; Restraint stress; Spatial memory; Water maze; Passive avoidance.

\section{INTRODUCTION}

Chronic stress has been proposed to compromise the function of the hippocampus, a region in the brain important for memory processing. ${ }^{1,2}$ Chronic exposure to stress steroids, known as glucocorticoids (GCs), makes hippocampal neurons vulnerable to GC such that over a life span, damage may eventually arise in the neurons. ${ }^{3,4}$ Though stress-induced memory impairments have been extensively studied, very few studies have looked into possible ways of preventing their deleterious effects.

I Department of Physiology, Melaka-Manipal Medical College, International Centre for Health Sciences, Manipal University - Manipal, Karnataka, India.

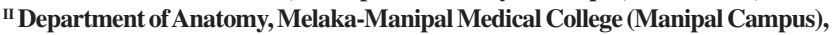
International Center for Health Sciences, Manipal University, Manipal, India. Email: raju.sureshkumar@manipal.edu Tel: 918202922646

Received for publication on August 04, 2009

Accepted for publication on September, 2009
Stress exerts detrimental effects on several cellular functions through impairment of antioxidant defenses, leading to oxidative damage, a process central to many diseases ${ }^{5}$. Antioxidant treatment has shown modulatory effects on brain free radicals in restraint rats. ${ }^{6}$ Vitamin $\mathrm{C}$ (ascorbic acid) is a water-soluble vitamin. It is not synthesized in the body and must be obtained through diet or supplementation. $\alpha$-tocopherol, ascorbic acid and reduced glutathione are important chain-breaking antioxidants responsible for scavenging free radicals and suppression of peroxidation in the cytosol and membrane of the cell. ${ }^{7,8}$ Ascorbic acid has been evaluated for its various pharmacological activities. It has been reported that ascorbic acid protects intestinal mucosa from ischemia. ${ }^{9}$ Ascorbic acid provided protection to young mice from scopolamine and diazepam-induced memory impairment. ${ }^{10}$ Previous studies have reported that intraperitoneal injections of ascorbic acid minimized oxidative stress in blood, liver, and muscles of 
rats with streptozotocin-induced diabetes. ${ }^{11}$ Though various studies on ascorbic acid are found in the literature, effects on spatial memory during restraint stress and the role in memory retention are not well addressed. It is well-known that chronic restraint stress impairs acquisition and retention of spatial memory tasks in rats. ${ }^{12}$ The present study was designed to investigate the effects of orally administered ascorbic acid in the prevention of restraint stress-induced memory deficits by using the Morris water maze and passive avoidance tests.

\section{MATERIALS AND METHODS}

\section{Animals}

Adult male Wistar rats (3 months old) weighing 200-250 $\mathrm{g}$ were used in the study. A stock of animals was obtained from the institutional animal facility after careful selective breeding. Animals belonging to six different litters (the average litter size being ten) were used in this study. Each litter had both male and female rats. We chose only male rats for this study. Animals from each of the six different litters were uniformly distributed by random allocation to each of the experimental groups used in this study. Three rats were maintained in each cage to minimize the stress caused by overcrowding. Rats were housed in polypropylene cages, $(22.5 \times 35.5 \times 15 \mathrm{~cm})$. All animals were maintained in a 12:12-hr light: dark environment, in an air conditioned room $\left(24 \pm 1^{\circ} \mathrm{C}\right)$ of the institutional animal facility. All rats were provided with water and food ad libitum, except during the stressing procedure. All procedures for the treatment of animals were approved by the research committee of the institute and were done in accordance with the Guide for Care and Use of Laboratory Animals published by the United States National Institutes of Health (NIH Publication No. 85-23, revised 1985).

\section{Experimental Design}

The rats were divided into six groups $(n=6$ in each group) as follows: (i) Normal control (NC), remained undisturbed in the home cage throughout the experimental period; (ii) Ascorbic acid treatment (AA), these rats were fed with an oral dose of $100 \mathrm{mg} / \mathrm{kg} / \mathrm{b}$.wt of ascorbic acid daily for 21 days; (iii) Vehicle control (V), these rats were fed with equivolume of vehicle solution $(0.9 \%$ sodium chloride solution) orally for 21 days; (iv) Restraint stress (S), these rats were stressed in wire mesh restrainers, $6 \mathrm{hrs} /$ day, for 21 days; (v) Restraint stress + vehicle $(\mathrm{S}+\mathrm{V})$, these rats were fed with an equivolume of vehicle solution $(0.9 \%$ sodium chloride solution) daily and stressed for $6 \mathrm{hrs} / \mathrm{day}$, for 21 days; and (vi) Restraint stress + ascorbic acid treatment (S $+\mathrm{AA}$ ), these rats were fed with an oral dose of $100 \mathrm{mg} /$ $\mathrm{kg} / \mathrm{b} . \mathrm{wt}$ of ascorbic acid daily and stressed for $6 \mathrm{hrs} / \mathrm{day}$, for 21 days. At the end of 21 days, rats in all groups were subjected to memory tests using passive avoidance apparatus and Morris water maze.

\section{Stress Procedure}

Rats were subjected to restraint stress in a wire mesh restrainer, 6 hours per day for 21 days. ${ }^{12}$ The stress procedure was carried out in the institutional animal facility throughout the experimental period between 10 am and $4 \mathrm{pm}$. The wire mesh restrainer had a wooden base and stainless steel wire mesh restrainer hinged to the base. A restrainer with dimensions of $8 \mathrm{~cm}(\mathrm{~L}) \times 4 \mathrm{~cm}(\mathrm{~B}) \times 4 \mathrm{~cm}(\mathrm{H})$ was used in this experiment.

\section{Ascorbic acid}

Ascorbic acid (Merck, India) was administered daily with an oral dose of $100 \mathrm{mg} / \mathrm{kg}$ body weight/day for 21 days. This dose was selected based on a previous study. ${ }^{13}$ Ascorbic acid was dissolved in isotonic sodium chloride solution (Merck, India) to achieve the desired concentration. Since ascorbic acid is highly sensitive to degradation, solutions were prepared each day, just before the beginning of the experiment. The volume given was adjusted for the weight of each rat so that all animals received a dose of $100 \mathrm{mg} / \mathrm{kg}$ body weight. Administration of ascorbic acid was done just before restraining the animals. The gavage procedure was carried out in the same animal facility where the animals were housed.

\section{Behavioral Tests}

\section{a) Passive avoidance test}

To test memory retention, rats were subjected to a passive avoidance test. ${ }^{14}$ The apparatus had two compartments: a large compartment and a small compartment. The rectangular, larger compartment had a $50 \times 50 \mathrm{~cm}$ grid floor and wooden walls of $35 \mathrm{~cm}$ height. It had a roof that could be opened or closed. One of the walls contained a $6 \times 6 \mathrm{~cm}$ opening connecting the larger compartment to a dark smaller compartment. The smaller compartment was constructed with wooden walls of $15 \mathrm{~cm}$ height and a ceiling that could be opened or closed, and a $15 \times 15 \mathrm{~cm}$ electrifiable grid floor connected to a constant current stimulator. The passage between the two compartments was closed with an opaque guillotine door.

The larger compartment was illuminated with a $100 \mathrm{~W}$ 
bulb placed $150 \mathrm{~cm}$ above the center. The experiment included three parts: (i) exploration, (ii) aversive stimulation and learning, and (iii) retention test. During exploration, each rat was kept in the center of the larger compartment facing away from the entrance to the dark compartment. The door between two compartments was kept open. The rat was allowed to explore the apparatus (both larger and smaller compartments) for a period of three minutes. Each rat had three trial sessions. After the last exploration trial and as soon as the rat entered the dark compartment, the sliding door between the two compartments was closed and three foot shocks ( $50 \mathrm{~Hz}, 1.5 \mathrm{~mA}$, and $1 \mathrm{sec}$ duration) were given at five-second intervals. The ceiling was then opened and the rat was returned to its home cage. The retention test was carried out after 24 and 48 hours. During the retention test each rat was placed in the larger compartment for a maximum of three minutes. The time taken by each rat to enter the dark compartment was measured using a stopwatch. The latency time was recorded as 3 minutes for animals that did not enter the dark compartment within the given three minutes. Absence of entry to the dark compartment or a longer duration in the bright compartment indicated a positive response.

\section{b) Morris water maze test}

Spatial memory of the rats was tested using the Morris water maze test ${ }^{15}$. The water maze apparatus consisted of a water tank of 1.80 meters in diameter and $75 \mathrm{~cm}$ in depth, which was filled with water $50 \mathrm{~cm}$ deep $\left(24 \pm 1^{\circ} \mathrm{C}\right)$. Four points on the rim of the pool were designated as north $(\mathrm{N})$, south (S), east (E), and west (W), thus dividing the pool into four quadrants. There was a 4"x4" size platform placed $2 \mathrm{~cm}$ below the water surface in one of the quadrants, the target quadrant. Nontoxic white paint powder was added to the water just before the experiment to make the water opaque. To provide extra-maze cues for allowing the rats to develop a spatial map strategy, two black and white pictures were hung on the walls. Positions of the cues were kept unchanged throughout the experimental period.

\section{Acquisition phase}

The rats were trained in the water maze in eleven sessions on six consecutive days, two sessions on each day except on the first day in which only one session was given. During each training session the rats were placed in water so that they faced the wall of the pool. Each session consisted of four trials. The trials were always initiated from different positions in the tank. For each rat, the quadrant in which the platform was located remained constant, but the point of immersion into the pool varied between $\mathrm{N}, \mathrm{E}, \mathrm{S}$, and $\mathrm{W}$ so that the rat was not able to predict the platform location from the location at which it was placed into the pool. The rat was then given three minutes to search for the platform. Once the rat located the platform, it was permitted to remain on it for $10 \mathrm{~s}$. In each training session, the latency (time) to escape onto the hidden platform was recorded. If the rat was unable to find the platform within three minutes, it was guided to the platform and allowed to remain on it for $10 \mathrm{~s}$. After each trial the rats were removed, dried using a towel and put back to their home cages.

\section{Probe trial}

One day following completion of the last training session, each rat was subjected to a probe trial $(30 \mathrm{sec})$ in which there was no hidden platform. The rat was placed into the water. The time taken to reach the target quadrant, the distance traveled by the animal in target quadrant and the time spent in the target quadrant were measured. All the events were video recorded using a video camera (Sony color camera with F1.2 Lens, 0.7 Lux). The data were analyzed using Panlab Smart Version 2.5 video tracking software, Barcelona, Spain. Greater latency to reach the target quadrant and less time spent in the target quadrant suggested memory impairment.

\section{Data analysis}

Analysis of variance (ANOVA) with repeated measures followed by Bonferroni multiple comparison tests (post-hoc) were used to compare the performance of different groups of rats in Morris water maze training sessions. SPSS version 10 statistical software was used. The passive avoidance test and probe trial test of the water maze were analyzed using one-way analysis of variance (ANOVA) followed by Tukey's multiple comparison test (post-hoc) using Graph pad in stat software. In both sets of analyses, the significance level was fixed at $\mathrm{P}<0.05$.

\section{RESULTS}

\section{i. Passive avoidance test}

The passive avoidance test revealed impaired memory retention in the stressed rats. In animals that were pre-treated with ascorbic acid before the stress procedure, memory deficits were minimized significantly. The stressed and stressed + vehicle-treated rats displayed very short latencies to enter the dark compartment of the passive avoidance apparatus when compared to normal control /vehicle control /ascorbic acid treated /ascorbic acid + stressed rats during the $24 \mathrm{hr}$ retention test and the $48 \mathrm{hr}$ retention test (Fig.1). Normal rats treated with ascorbic acid showed significant 


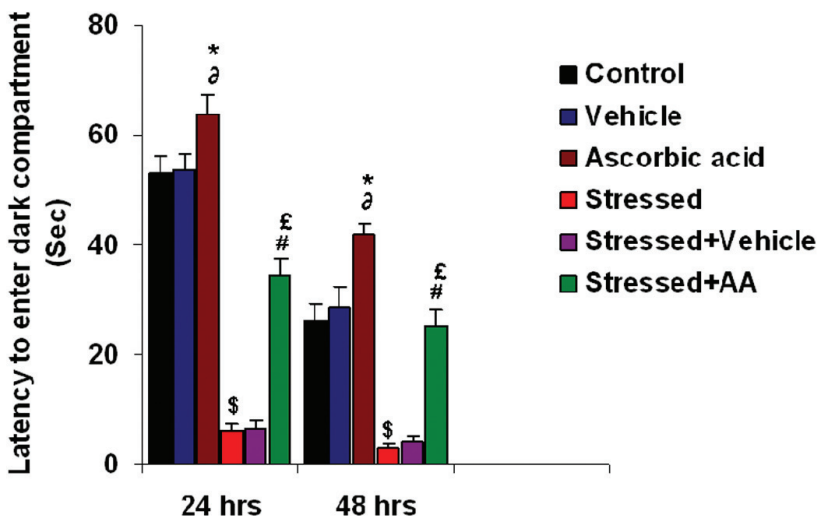

Figure 1 - Latency to enter the dark compartment of passive avoidance apparatus during memory retention test after 24 and $48 \mathrm{hr}$. Data represent mean $\pm \operatorname{SEM}(n=6)$. Normal control/vehicle control vs. stressed, $\$ \mathrm{P}<0.001$; stressed vs. stressed + ascorbic acid treated, \# P<0.001; stressed + vehicle vs. stressed + ascorbic acid treated, $\leq \mathrm{P}<0.001$; control vs. ascorbic acid treated, $\partial \mathrm{P}<0.01$; vehicle control vs. ascorbic acid, $* \mathrm{P}<0.05$; after $24 \mathrm{hrs}$ and $48 \mathrm{hrs,}$, one-way ANOVA, Tukey (post-hoc) improvement in memory retention during the $48 \mathrm{hr}$ retention test compared to normal and vehicle controls. The result of this test suggests the protective effects of ascorbic acid against stress-induced memory impairment.

\section{ii. Morris water maze test}

\section{Water maze performance during the training sessions}

Analysis of water maze training sessions during the six successive days revealed deficits in progressive learning in rats that subjected to restraint stress and those pretreated with vehicle solution prior to restraint stress (Fig. 2A). However, all rats of other groups began to identify the platform from the second day, and continued to retain their learning ability throughout the training period. At the end of the 6th day, results showed enhanced progressive learning in normal control/vehicle control/ascorbic acid
A

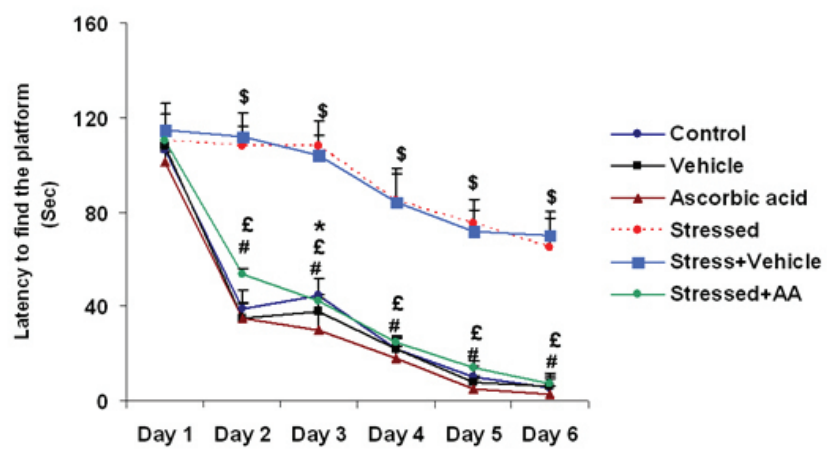

C

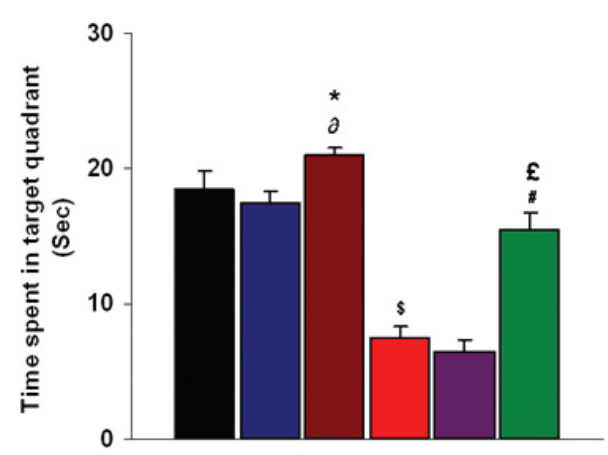

B
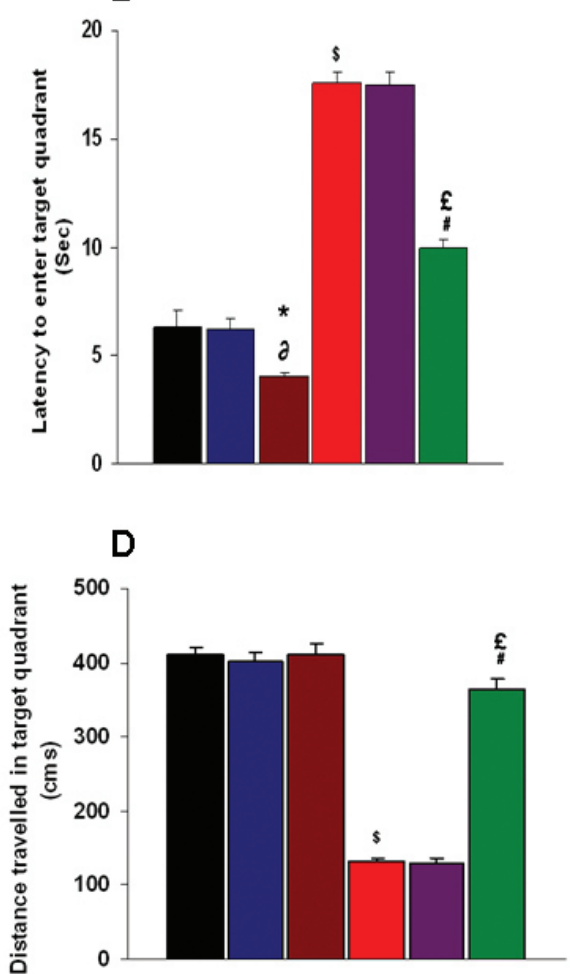

Figure 2 - A - Progressive learning of rats in different groups during six successive days of water maze training (Fig. 2A). Data represent mean \pm SEM $(\mathrm{n}=6)$. Normal control/vehicle control vs. stressed, $\$ \mathrm{P}<0.001$; stressed vs. stressed + ascorbic acid treated \# $\mathrm{P}<0.001$, stressed + vehicle vs. stressed+ ascorbic acid treated, $£ \mathrm{P}<0.001$; from day 2 to day 6 ; normal control/vehicle control vs. ascorbic acid treated, on day three, ${ }^{*} \mathrm{P}<0.05$; analysis of variance (ANOVA) with repeated measures followed by Bonferroni (post-hoc). B - Latency to enter the target quadrant during the probe trial. Data represent mean \pm SEM ( $n=6$ ). Normal control vs. stressed, \$ P <0.001; stressed vs. stressed + ascorbic acid treated, \# P $<0.001$; stressed+ vehicle vs. stressed+ ascorbic acid treated, $£ \mathrm{P}<0.001$; normal control vs. ascorbic acid treated, $\partial \mathrm{P}<0.01$; vehicle control vs. ascorbic acid treated, $* \mathrm{P}<0.01$; one-way ANOVA, Tukey (post-hoc). C - Time spent in the target quadrant during the probe trial. Data represent mean \pm SEM ( $\mathrm{n}=6$ ). Normal control/vehicle control vs. stressed, $\$$ $\mathrm{P}<0.001$; stressed vs. stressed + ascorbic acid treated \# $\mathrm{P}<0.001$, stressed + vehicle control vs. stressed + ascorbic acid treated, $£$ P $<0.001 ;$ normal control vs. ascorbic acid treated, $\partial \mathrm{P}<0.05$; vehicle control vs. ascorbic acid treated, ${ }^{*} \mathrm{P}<0.01$; one-way ANOVA, Tukey (post-hoc). D - Distance traveled in the target quadrant during probe trial test. All data represent mean \pm SEM $(n=6)$; normal control vs. stressed, P $<0.001$; stressed vs. stressed + ascorbic acid treated, $\mathrm{P}<0.001$; stressed+ vehicle vs. stressed+ ascorbic acid treated, $£ \mathrm{P}<0.001$; one-way ANOVA, Tukey test (post-hoc) 
treated and stressed +AA treated groups. The stressed and stressed + vehicle treated rats took longer to identify and to reach the hidden platform, indicating memory impairment. The progressive learning over days in these groups was much lower as compared to the other groups. Normal rats treated with ascorbic acid showed a significant increase in progressive learning on day three when compared to normal control and vehicle-treated rats. They displayed a comparatively shorter duration to reach the platform of the target quadrant. Supplementation with ascorbic acid during restraint stress exerted protective effects in rats. These results suggest a possible facilitation of progressive learning in ascorbic acid-treated animals during the stress period.

\section{Latency to enter the target quadrant during the probe trial}

The stressed rats and stressed + vehicle-treated rats took longer to reach the target quadrant (fig. 2B), whereas rats pre-treated with ascorbic acid before restraint stress and normal control, vehicle control and ascorbic acid-treated animals took less time to reach the target quadrant.

\section{Time spent in the target quadrant during probe trial}

Analysis of swimming performance during the probe trial evidenced that animals submitted to restraint stress spent significantly less time in the quadrant of the former platform position (Fig. 2C). Rats in the control group, vehicle group, ascorbic acid-treated group and stressed rats pretreated with ascorbic acid spent more time in the target quadrant.

\section{Distance traveled in the target quadrant during probe trial}

The stressed rats and stressed + vehicle-treated rats traveled a shorter distance in the target quadrant, whereas rats in all the other groups traveled a longer distance in the target quadrant (Fig. 2D). These results suggest possible protective effects of ascorbic acid in prevention of spatial memory impairment in rats.

Fig. 3 shows video tracking of the swim paths of one representative animal from each group during the probe trial test. The paths taken by normal control, vehicle control, ascorbic acid-treated and stressed + ascorbic acid-treated rats were largely confined to the trained target quadrant, whereas the paths taken by stressed and stressed + vehicle-treated rats appeared as circular patterns confined more to quadrants other than target quadrant.

\section{DISCUSSION}

Restraint stress is known to impair spatial learning and memory. ${ }^{12}$ In the present study, animals receiving restraint stress demonstrated memory impairment that was evident in
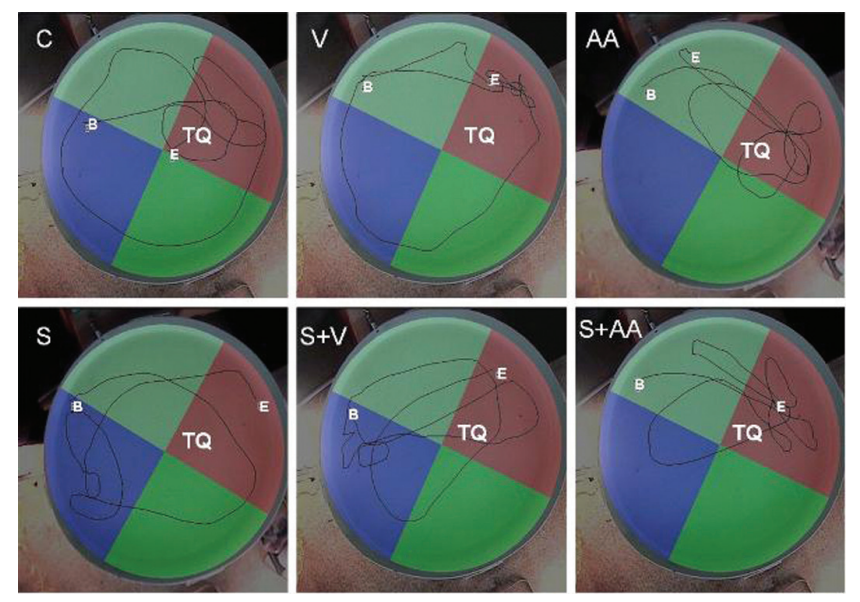

Figure 3 - Video tracking of swim paths taken by a representative rat in each group with respect to time spent in the former location of the platform during the probe trial. Normal control (NC), vehicle control (V), ascorbic acid (AA), stressed (S), stressed+ vehicle $(\mathrm{S}+\mathrm{V})$ stressed + ascorbic acid treated (S+AA) rats. Note that stressed rats spent less time in the target quadrant (TQ), unlike stressed + ascorbic acid-treated rats, which spent most of their time there. B- beginning, E- end of the tracking

the Morris water maze test and passive avoidance tests. Such memory impairment was attenuated by oral administration of ascorbic acid during the stress period.

\section{Ascorbic acid, stress and memory}

Parle and Dhingra (2003) reported that ascorbic acid enhanced memory in aged mice and reversed memory impairment in scopolamine- and diazepam-treated young mice when administered intraperitoneally at $60 \mathrm{mg} / \mathrm{kg}$ body weight. ${ }^{10}$ In our study, normal rats treated with ascorbic acid showed a significant enhancement in memory. We could also demonstrate a protective effect of ascorbic acid in preventing restraint stress-induced memory loss in rats. The mechanism of such an action of ascorbic acid may be attributed to its neuroprotective role. ${ }^{16}$

\section{Oxidative stress and the brain}

If an equilibrium between the rate of free radical formation and neutralization is not maintained, oxidative stress results. ${ }^{17}$ Chronic stress is known to cause memory impairments. ${ }^{12}$ The neural basis for such an impairment has been demonstrated to be due to injury in the hippocampus. ${ }^{18}$ Neuronal damage in this region may also be mediated through increased glucocorticoid production and excitotoxicity. ${ }^{19}$ The central nervous system is vulnerable to free radical damage because of the brain's high oxygen consumption, its abundant lipid content, and the relative paucity of antioxidant enzymes as compared to other tissues. ${ }^{20}$ The brain is deficient in oxidative defense 
mechanisms and hence is at greater risk of damage mediated by reactive oxygen species (ROS), resulting in molecular and cellular dysfunction. ${ }^{21}$ Oxidative stress has been demonstrated to be related to the pathophysiologic mechanisms involved in brain injury in various common neurodegenerative disorders, including Parkinson's, Alzheimer's and Huntington's diseases. Stress results in oxidative stress and also decreases antioxidant defense status in the brain, which may form the basis for impaired memory. ${ }^{22,23}$ Thus, in the present study, the memory deficits observed during restraint stress might have arisen as a result of increased free radical formation and subsequent oxidative injury to neurons.

\section{Mechanism of ascorbic acid in restraint stress}

Oxygen free radicals and other products of oxidative metabolism have been shown to be neurotoxic. ${ }^{24}$ Restraint stress may cause generation of free radicals and result in oxidative injury to brain. ${ }^{13}$ Studies have shown that dehydroascorbic acid, the oxidized form of ascorbic acid, enters the brain by means of facilitated transport. ${ }^{25}$ GLUT-1 transporter present on the endothelial cells of the blood-brain barrier helps in the transport of glucose and dehydroascorbic acid into the brain. ${ }^{26}$ Oral administration of ascorbic acid during restraint stress may have resulted in the entry of high levels of dehydroascorbic acid into the rat brain, which could protect neurons from the deleterious effects of free radicals. In conclusion, ascorbic acid, a simple water soluble vitamin, may be effectively used in stress-related neurological disorders where cognition is impaired.

\section{ACKNOWLEDGEMENTS}

The authors would like to thank Mr. Vinod Pallath from the Department of Microbiology, Melaka- Manipal Medical College (Manipal Campus) for the technical assistance provided in the statistical analysis of this study.

\section{REFERENCES}

1. Eichenbaum H. How does the brain organize memories. Science. 1997;277:330-32

2. O'Keefe J, Nadel L, The hippocampus as a cognitive map. Oxford, UK Clarendon Press; 1978.

3. Sapolsky RM, Krey LC, McEwen BS. The neuroendocrinology of stress and aging: the glucocorticoid cascade hypothesis. Endocrine Reviews. 1986;7:284-301.

4. Sapolsky RM. Stress, the aging brain, and the mechanisms of neuron death. Cambridge, MIT Press, 1992.

5. Torres RL, Torres ILS, Gamaro,GD, Fontella FU, Silveira PP, Moreira JSR, et al. Lipid peroxidation and total radical-trapping potential of the lungs of rats submitted to chronic and subchronic stress. Braz. J. Med. Biol. Res. 2004;37:185-92.

6. Zaidi SM, Banu N. Antioxidant potentials of vitamin A, E, and C in modulation oxidative stress in rat brain. Clin.Chim Acta. 2004;340:22933.

7. Niki E. Interaction of ascorbate and alphatocopherol. Ann N Y Acad Sci.1987;498:186-99

8. Mann GV, Newton P. The membrane transport of ascorbic acid. Ann N Y Acad Sci. 1975;258:243-52.

9. Higa OH, Parra ER, Ab'Saber AM, Farhat C, Higa R, Capelozzzi VL. Protective effects of ascorbic acid pretreatment in a rat model of intestinal ischemia-reperfusion injury: a histomorphometric study. Clinics. 2007:62:315-20.

10. Parle M, Dhingra D. Ascorbic acid: a promising memory enhancer in mice. J Pharmacol Sci. 2003;93:129-35.
11. Cay M, Naziroglu M, Simsek H, Avdilek N, Akskal M, Demirci M. Effects of intraperitonially administered vitamin $\mathrm{C}$ on oxidative defense mechanism in rats with diabetes induced by streptozotocin. Res Exp Med. 2001;200:205-13

12. Sunanda, Rao BSS, Raju TR. Chronic restraint stress impairs acquisition and retention of spatial memory task in rats. Current Science. 2000;79:1581-84

13. Venkataraman P, Muthuvel R, Krishnamoorthy G, Arun Kumar A, Sridhar M, Srinivasan N, et al. PCB (Aroclor 1254) enhances oxidative damage in rat brain regions: Protective role of ascorbic acid. Neurotoxocology. 2007;28:490-8

14. Bures J, Buresova A, Huston JP. Passive avoidance. In. Techniques and basic experiments for the study of brain and behavior; 2nd revised and enlarged edition. Elsevier science publishers, Amsterdam New York; 1983.p.148-60.

15. Rudi DH, Peter PD. Applications of Morris water maze in the study of learning and memory. Brain Research Reviews. 2001;36:60-90.

16. Rice ME. Ascorbate regulation and its neuroprotective role in the brain. Trends Neurosci. 2000;23:209-16

17. Sies H. Oxidative stress: From Basic Research to Clinical Application. Am. J. of Med. 1991;91:31S-38S.

18. Sunanda, Meti BL, Raju TR. Entorhinal cortex lesioning protects hippocampal CA3 neurons from stress-induced damage. Brain Research. 1997;770:302-6.

19. McEwen BS. Physiology and neurobiology of stress and adaptation: central role of the brain. Physiol.Rev.2007; 87:873-904. 
20. Skaper SD, Floreani M, Ceccon M, Facci L, Giusti P. Excitotoxicity oxidative stress, and the neuroprotective potential of melatonin. Ann N Y Acad Sci. 1999;890:107-18.

21. Gupta YK, Gupta M, Kohli K. Neuroprotective role of melatonin in oxidative stress vulnerable brain. Indian Journal of Physiology and Pharmacology. 2003;47:373-86.

22. Mates JM, C. Perez-Gomez C, Castro N I. Antioxidant enzymes and human diseases, Clin. Biochem.1999; 32:595-603.

23. Onodera K, Omoi NO, Fukui K, Hayasaka T, Shinkai T,Suzuki S, et al. Oxidative damage of rat cerebral cortex and hippocampus, and changes in antioxidative defense system caused by hyperoxia. Free Radical Research. 2003;37:367-72.
24. Sayre LM, Zagorski MG, Surewicz WK, Krafft GA, Perry G. Mechanisms of neurotoxicity associated with amyloid beta deposition and the role of free radicals in the pathogenesis of Alzheimer's disease: a critical appraisal. Chem Res Toxicol. 1997;336:1216-22.

25. Agus DB, Gambhir SS, Pardridge WM, Spielholz C, Baselga J, Vera J $\mathrm{C}$, et al. Vitamin C crosses the blood-brain barrier in the oxidized form through the glucose transporters. J Clin Invest. 1997;100:2842-8.

26. Huang J, Agus DB, Winfree CJ, Kiss S, Mack WJ, Mctaqqart RA, et al. Dihydroascorbic acid, a blood-brain barrier transportable form of Vitamin C, mediates potent cerebroprotection in experimental stroke. Proceedings of National Academy of Sciences USA. 2001;98:11720-4. 
